\title{
FERMI LAT OBSERVATION OF CENTAURUS A RADIO GALAXY
}

\author{
N. V. SAHAKYAN \\ Dipartimento di Fisica, Università di Roma La Sapienza \\ Piazzale Aldo Moro 5, I-00185, Roma, Italy \\ ICRANet, Piazzale della Repubblica 10 \\ I-65122 Pescara, Italy \\ Institute for Physical Research, NAS of Armenia \\ Ashtarak-2, 0203, Armenia \\ narek@icra.it
}

\begin{abstract}
The results of analysis of approximately 3 year gamma-ray observations (August 2008July 2011) of the core of radio galaxy Centaurus A with the Fermi Large Area Telescope (Fermi LAT) are presented. Binned likelihood analysis method applying to the data shows that below several $\mathrm{GeV}$ the spectrum can be described by a single power-law with photon index $\Gamma=2.73 \pm 0.06$. However, at higher energies the new data show significant excess above the extrapolation of the energy spectrum from low energies. The comparison of the corresponding Spectral Energy Distribution (SED) at GeV energies with the SED in the $\mathrm{TeV}$ energy band reported by the H.E.S.S. collaboration shows that we deal with two or perhaps even three components of gamma-radiation originating from different regions located within the central $10 \mathrm{kpc}$ of Centaurus A.

The analysis of gamma-ray data of Centaurus A lobe accumulated from the beginning of the operation until November 14, 2011 show extension of the HE gamma-ray emission beyond the WMAP radio image in the case of the Northern lobe [9]. The possible origins of gamma-rays from giant radio lobes of Centaurus A are discussed in the context of hadronic and leptonic scenarios.
\end{abstract}

Keywords: Centaurus A; gamma-rays; radio galaxy; likelihood analysis.

\section{Introduction}

At a distance of $3.8 \mathrm{Mpc}$ the peculiar elliptical radio galaxy NGC 5128 (Centaurus A) is the nearest active radiogalaxy and one of the best studied extragalactic objects. It is often treated as a prototype Fanaroff-Riley Class I [1] low-luminosity radio galaxy. With the size of $\approx 600 \mathrm{kpc}$, it has an interesting structure: giant radio lobes with an angular extent of $10^{\circ}$ oriented primarily in the north-south direction, a relativistic jet extending to kpc scales, inner double lobes, and a compact core. The observations of hot thermal X-ray emission, show that the inner lobes are a result of the current nuclear activity. It is generally believed that the energy is extracted from the central black hole of mass $\approx 10^{8} \mathrm{M}_{\odot}$. 
Recently the Fermi LAT collaboration reported the detection of high energy gamma-rays from both the north and south giant radio lobes [2]. It has been suggested that this radiation is the result of inverse Compton scattering of cosmic microwave background photons and the extragalactic background light photons by in situ accelerated electrons [2]. The central inner part (the core) of the radiogalaxy was previously detected by EGRET (Energetic Gamma-Ray Experiment Telescope) with marginal statistical significance of about $3 \sigma$ and energies up to several hundreds $\mathrm{MeV}$ [3]. The core of Cen A was a target of long-term observations by the H.E.S.S. (High Energy Stereoscopic System) Imaging Atmospheric Cherenkov Telescope Array. These observations resulted in detection of gamma-rays were detected up to energies of $5 \mathrm{TeV}$ [4]. According to the HESS data, the power-law photon index of the source is $\Gamma=2.73 \pm 0.45_{\text {stat }} \pm 0.2_{\text {syst }}$, and integral flux above $250 \mathrm{GeV}$

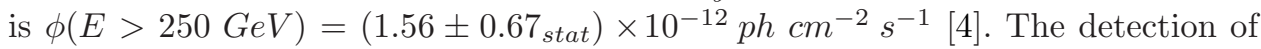
Centaurus A by EGRET was confirmed by Fermi LAT telescope, earlier with the three month of data [5] and later on with the 10 month of all-sky survey data. The Fermi LAT data show that the power-law photon index for the energies between $200 \mathrm{MeV}$ and $30 \mathrm{GeV}$ is $\Gamma=2.67 \pm 0.10_{\text {stat }} \pm 0.08_{\text {sys }}$ and the flux extrapolated down to $>100 \mathrm{MeV}$ is $\left(1.50 \pm 0.25_{\text {stat }} \pm 0.37_{\text {syst }}\right) \times 10^{-7} \mathrm{ph} / \mathrm{cm}^{2} \mathrm{~s}[6]$.

In this paper we discuss $i$ ) the results of analysis of the Fermi LAT data accumulated over the last 3 years (from August 4th 2008 to July 7th 2011), with the objective to study of spectral properties of the gamma-ray emission from the core of Centaurus A and ii) the origin of gamma-ray emission from the lobes of Centaurus A.

\section{Centaurus A Core}

Data selection and analysis: The data used for present studies of the core of radio galaxy Cen A is based on approximately 3 years of continuous sky survey over the period from August 4th 2008 to July 7th 2011 (corresponding to Mission Elapsed Times (MET) 239557420-331755684). For the analysis photons within a rectangular Region of Interest (ROI) of size $14^{\circ} \times 14^{\circ}$ centered on the position of Right Ascension(RA) and Declination (Dec) (RA, Dec) $=(201.47,-42.97)$ [7]. The energy band covers from $200 \mathrm{MeV}$ to $50 \mathrm{GeV}$. For the lower energy bound one sets the limit of $200 \mathrm{MeV}$ since at lower energies the effective area of Fermi LAT is rapidly reduced, and the angular resolution becomes too large. On the other hand, the limit of photon statistics determines the upper bound of $50 \mathrm{GeV}$. Also, a zenith angle cut of $<105^{\circ}$ was introduced in order to exclude the albedo atmospheric $\gamma$ rays that can be a significant source of background. For the data analysis, the Fermi LAT Science Tools version v9r23p1 was used with the Instrument Response Function (IRF) P6_V11_DIFFUSE. The galactic and extragalactic components as well as the residual instrumental backgrounds are modeled using the Fermi LAT standard diffuse background models gll_iem_v02_P6_V11_DIFFUSE.fit 


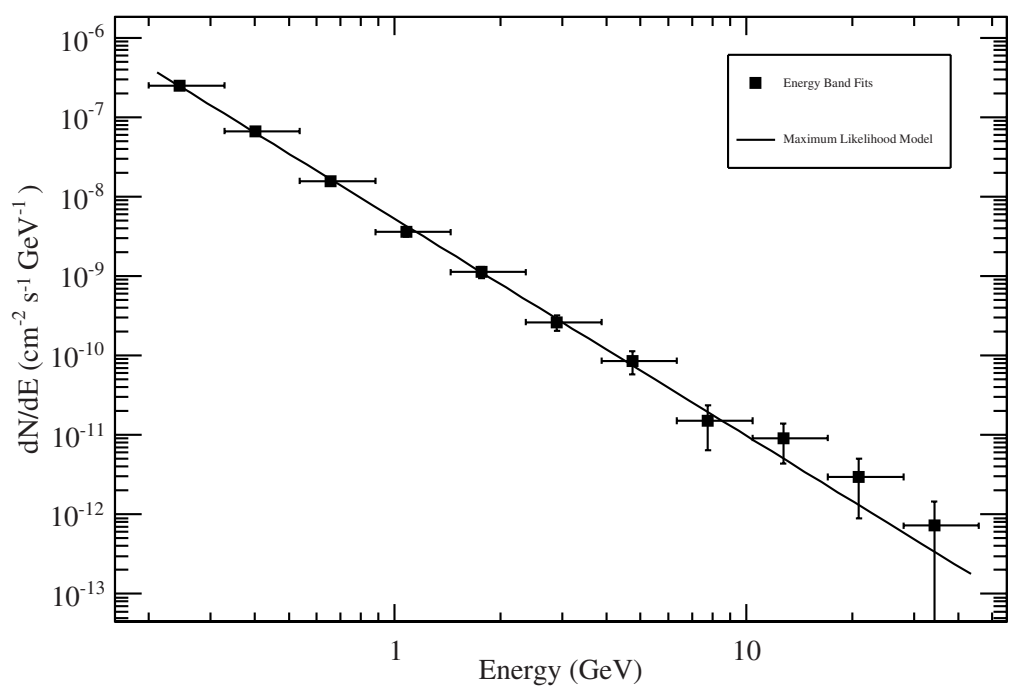

Fig. 1. The average LAT $(>200 \mathrm{MeV})$ spectrum of Centaurus A core for the observation time between (239557420 - 331755684) MET.

and isotropic_iem_v02_P6_V11_DIFFUSE.txt ${ }^{\mathrm{a}}$. For both models, the overall normalization is kept as a free parameter. (for more detailed description of the modeling of region including CenA galaxy see [8] ).

At the data preparation we use gtselect tool in order to make cuts based on events data file such as time, energy, position, zenith angle, instrument coordinates and event class, and gtmktime tool to make cuts based on the spacecraft file and updates the good time interval extension.

Spectral analysis: The results of the binned likelihood analysis gives for the Centaurus A core $\mathrm{TS}=990$ which corresponds to the detection significance $\approx 31 \sigma$. The power-law fit gives for the photon index $\Gamma=2.73 \pm 0.06$ within the $200 \mathrm{MeV}$ and $50 \mathrm{GeV}$ energy interval and the total flux $(1.63 \pm 0.14) \times 10^{-7} \mathrm{ph} \mathrm{cm}^{-2} \mathrm{~s}^{-1}$ above $200 \mathrm{MeV}$ [8].

Time Variability: For the investigation of variations of the flux and the photon index from the core of Centaurus A the time period from 4th 2008 to July 7th 2011 has been analyzed. In Figure 2 the plot of variation of flux (upper panel) and photon index of the core (lower panel) are presented as a function of time in Modified Julian Days (MJD). We use standard $\chi^{2}$ test, by the definition of [10], resulting to $\chi^{2}=19\left(\chi^{2} / D O F=0.56\right)$. The results presented in Figure 2 show that the time behavior of the core of Centaurus. Although the gamma-ray lightcurve shows possible variation of the gamma-ray flux on scales of 1 month, within a factor of two or so, unfortunately the poor statistics does not allow a definite conclusion in this regard. It should be noted that the observations of the core of Centaurus A with 

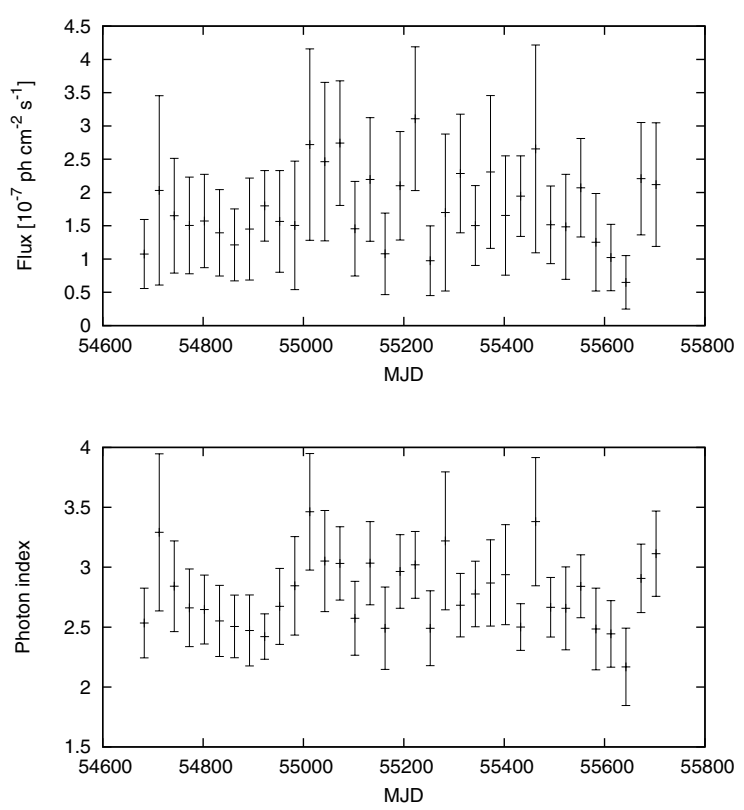

Fig. 2. The temporal variation of $\gamma$-ray flux and the photon index for the period from August 4th 2008 to July 7th 2011 with 30 day size of bins

the H.E.S.S. telescope array also did not reveal significant variability at very high energies [4]. For comparison, the $\mathrm{GeV}$ and $\mathrm{TeV}$ gamma-ray fluxes of blazars show strong (up to a factor of 10) variability both on short (mins) and long (months) timescales.

Spectral Energy Distribution: In Figure 3 we show the obtained Spectral Energy Distribution (SED) for the core of Cen A and comparison with the H.E.S.S. data. The angular resolutions of both the H.E.S.S. array of about $0.1^{\circ}$ (or worse) and the Fermi LAT $0.1^{\circ}-1^{\circ}$ (depending on energy) correspond to the linear size of the gamma-ray emitting region(s) of about $5 \mathrm{kpc}$ or larger. The $5 \mathrm{kpc}$ region contains several prominent components including the sub-pc and $\mathrm{kpc}$ scale jets, inner lobes, the compact core with suspected $\approx 10^{8} \mathrm{M}_{\odot}$ black hole inside, the elliptical galaxy itself, etc. All these regions are potential gamma-ray emitters. The limited angular resolutions of gamma-ray detectors do not allow to localize the regions of gammaray production. On the other hand the results from [8] indicate that most likely we deal with one or perhaps more gamma-ray sources contributing to the emission in different energy bands. The results of analysis shown in Figure 3 indicate that significant excess found in this paper between 5 and $30 \mathrm{GeV}$ (above the extrapolation of the flux from lower energies) has more general implication. Most likely this is a new component which perhaps can account also for the gamma-ray fluxes below 1 TeV reported by the H.E.S.S. collaboration [4] (first 3 points in Figure 3). Hopefully more observation data from Fermi LAT and H.E.S.S. should be able to provide 


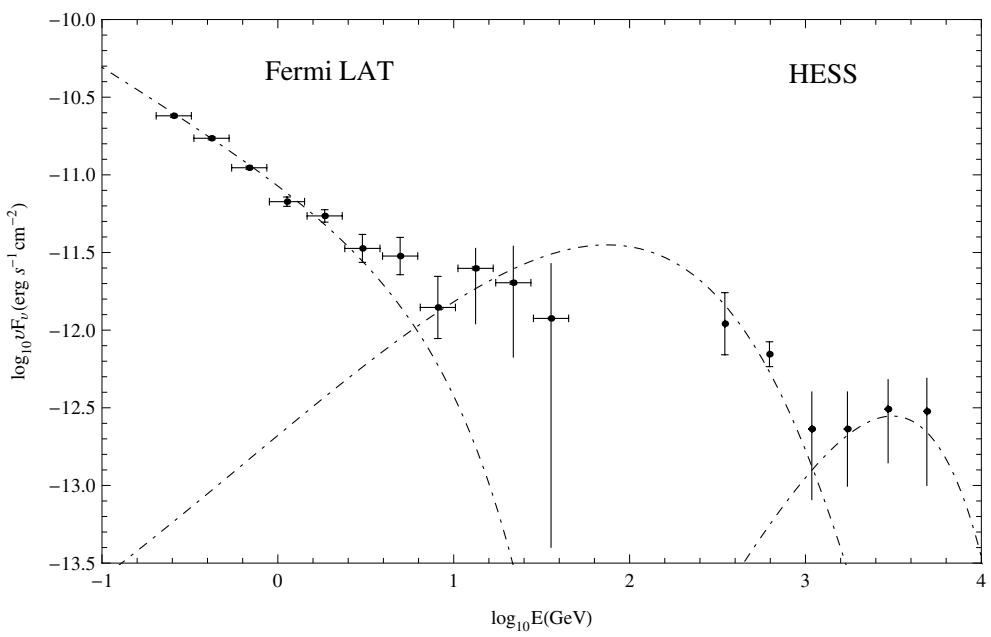

Fig. 3. Spectral Energy Distribution (SED) of the core of Centaurus A core and comparison with the HESS data. The dashed curves illustrate the possible three components of gamma-radiation in the core.

deeper understanding of $\gamma$-rays emission production sites and particle acceleration and radiation mechanisms in the core of Centaurus A.

\section{Centaurus a Lobes}

The analysis of Fermi LAT data of giant radio lobes of Centaurus A extension of the HE gamma-ray emission beyond the WMAP radio image in the case of the Northern lobe has been found [9]. Using radio (WMAP) and Fermi-LAT data from [9] the SED of Centaurus A lobe are discussed in the context of leptonic and hadronic scenario. We discuss the SED implications for a minimum and a maximum epoch time $\mathrm{t}$ of $\mathrm{t}_{\min }=10^{6} \mathrm{yr}$ and $\mathrm{t}_{\max }=8 \times 10^{7} \mathrm{yr}$.

In Figure 4 the SED of the North and South lobe is shown for the minimum epoch time $t_{\min }=10^{6}$ yr and $\gamma_{\max }=9.5 \times 10^{4}$. In this case the resulting gammaray flux can only described if one include Extragalactic Background Light (EBL) photons in addition to CMB photons (in Figure 4 the dashed line is inverse-Compton scattering of only $\mathrm{CMB}$ photons and the solid line is for total (CMB+EBL) photon field).

Figure 5 shows a representation of the SED for the maximum epoch time $t_{\max }$ $=8 \times 10^{7} \mathrm{yr}$ with a maximum electron Lorentz factor $\gamma_{\max }=2.5 \times 10^{6}$ and $1.5 \times 10^{6}$ for the North lobe and the South lobe, respectively. In this case the contribution by inverse-Compton scattering of $\mathrm{CMB}$ photons alone is sufficient to account for the observed HE spectrum (see the solid line in Figure 5). If the epoch time t exceeding $\mathrm{t}_{\max }=810^{7} \mathrm{yr}$, the high energy part of the SED would no longer be consistent with the data (see the dashed line in Fig. 9 for $\mathrm{t}=10^{8} \mathrm{yr}$ ). 

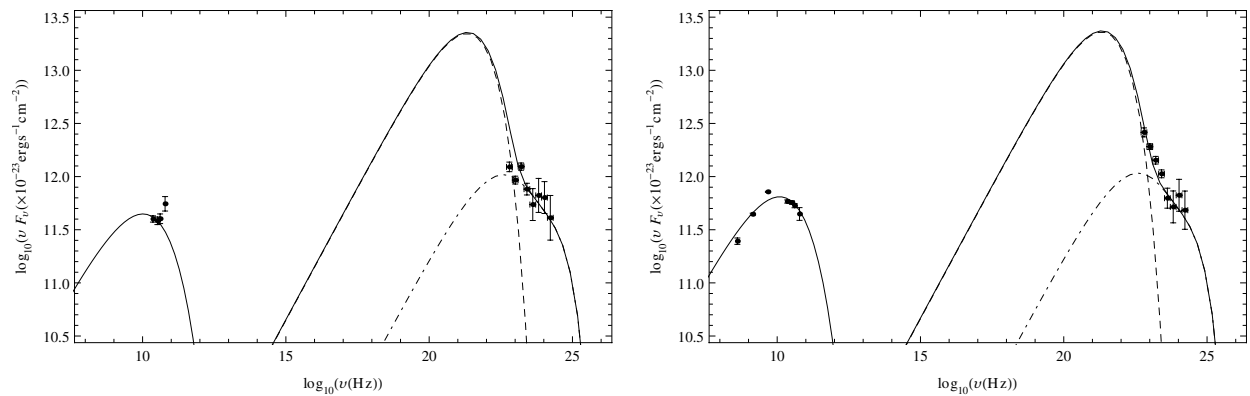

Fig. 4. Synchrotron and inverse Compton fluxes for $t=10^{6} \mathrm{yr}$. The radio data for the South lobe are from $\left[{ }^{11}\right]$ (sum of Region 4 and Region 5 in their Table 1), while the radio data for the North region are from the WMAP analysis in this paper. The mean magnetic field value $B$ used for the North and the South lobe is $0.38 \mu G$ and $0.45 \mu G$, respectively.
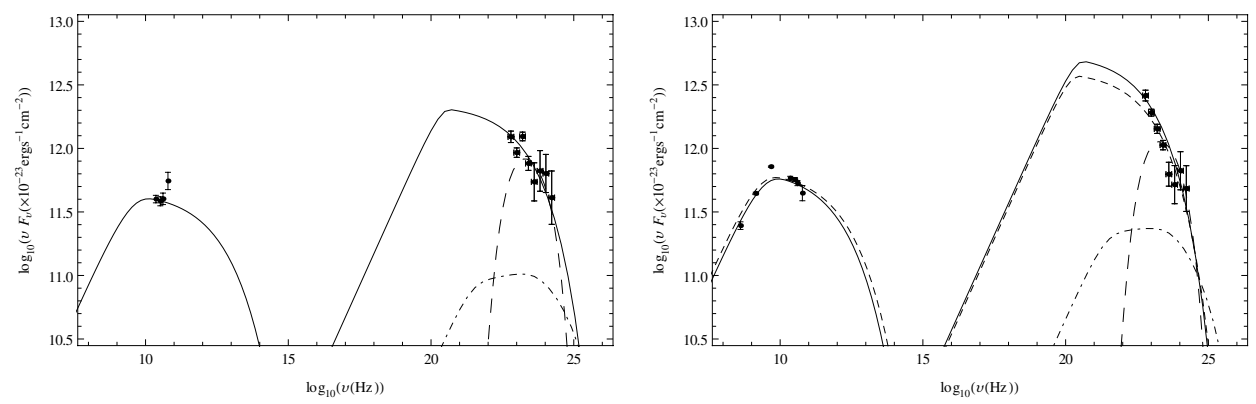

Fig. 5. Synchrotron and inverse Compton fluxes for $t=8 \times 10^{7} \mathrm{yr}$. The mean magnetic field value $B$ for South lobe and the $\gamma$-ray excess region in North is $1.17 \mu G$ and $0.91 \mu G$ respectively.

\section{Hadronic origin?}

Once protons get efficiently injected, they are likely to remain energetic since the cooling time for $p p$-interactions is $t_{p p} \approx 10^{15}\left(n / 1 \mathrm{~cm}^{-3}\right)^{-1} \mathrm{~s}$. High-energy protons interacting with the ambient low density plasma, then can produce daughter mesons and the $\pi^{0}$ component decays into two gamma-rays. High energy gamma ray data allow one to derive an upper limit on the energetic protons contained in the lobes of Centaurus A. We use $n=10^{-4} \mathrm{~cm}^{-3}$ and power-law form of protons for the model representation shown in Figure 5 (the dashed line). In both lobes, the power-law index of the proton population is $\alpha=2.1$, and the high-energy cut-off is $E_{\max } \simeq 55$ $\mathrm{GeV}$. The maximum total energy $W_{p}$ is proportional to the gas number density $n$, so that $W_{p} \simeq 10^{61}\left(n / 10^{-4} \mathrm{~cm}^{-3}\right)^{-1} \mathrm{erg}$, obtained here, should be considered as an upper limit.

\section{Summary}

The gamma-ray Fermi LAT observations of the core of radiogalaxy Centaurus A accumulated over three years (from August 4th 2008 to July 7th 2011) have been 
analyzed. For the energy range from $200 \mathrm{MeV}$ to $50 \mathrm{GeV}$ the total flux of the

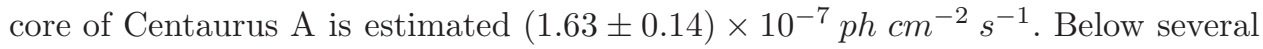
$\mathrm{GeV}$ the energy spectrum can be represented by a single power-law with photon index $\Gamma=2.73 \pm 0.06$, however above $5 \mathrm{GeV}$ the spectrum shows significant excess which can be a result of a new additional component of radiation. No statistically significant variability has beed found on scales of months.

The results of detailed study of the modeling of SED of both lobes imply a finite age $<10^{8} \mathrm{yr}$ of the lobes and a mean magnetic field strength $B \leq 1 \mu G$. While for lobe lifetimes of the order of $8 \times 10^{7} \mathrm{yr}$, inverse-Compton up-scattering of CMB photons alone would be sufficient to account for the observed HE spectrum, up-scattering of EBL photons is needed in the case of shorter lobe lifetimes.

\section{References}

1. Israel, F. P., Astronomy and Astrophysics Reviews, 8 237, 1997

2. Abdo, A., et al. (Fermi-LAT collabaration), Science, 328, 725, 2010a.

3. Hartman, R. C., et al. ApJS, 123, 79, 1999.

4. Aharonian, F., et al. ApJ, 695, L40, 2009.

5. Abdo, A., et al. (Fermi-LAT collaboration), ApJS, 183, 46, 2009.

6. Abdo, A., et al. (Fermi-LAT Collaboration), ApJ 719, 1433, 2010b.

7. Ma, C., et al. AJ,116,516 1998.

8. N.V. Sahakyan, High energy $\gamma$-radiation from the core of radio galaxy Centaurus A, 2012, Accepted for publication in Astrophysics.

9. Yang, R.Z., Sahakyan, N. et al., arXiv1201.1217Y, 2012, submitted to Astronomy and Astrophysics.

10. Abdo A. et al., ApJS, 188, 405, 2010d

11. Hardcastle M. et al., MNRAS, 393, 1041, 2009 